normal or subnormal levels of serum vitamin $B_{12}$ after partial gastrectomy are much more common than those with overt megaloblastic anaemia. ${ }^{6}$ The vitamin- $B_{12}$ deficiency is due to a failure of secretion of intrinsic factor as a consequence of atrophic gastritis in the gastric remnant, ${ }^{7}$ and only in a small minority of cases is a blind-loop syndrome to blame. In the Scandinavian cases of " myelopathy" the average time elapsing between operation and the onset of neurological symptoms was more than 10 years. Such a long latent interval need occasion no surprise if vitamin- $B_{12}$ deficiency is responsible, for impairment of intrinsic factor secretion would take time to develop from atrophic gastritis in the gastric remnant, and the hepatic store of vitamin $\mathbf{B}_{12}$ would also have to become depleted.

The recent report from Birmingham ${ }^{2}$ suggests that neurological disease after gastrectomy may be more complex than was thought at first and that vitamin- $B_{12}$ deficiency may not invariably be responsible. Serum levels of vitamin $B_{12}$ were below normal, and there was evidence of malabsorption of the vitamin in most patients, but some had normal serum levels and normal absorption.

Clinically all patients complained of difficulty in walking, with unsteadiness and muscular weakness; most also had paraesthesiae or numbness and a feeling of malaise or depression. Muscle weakness and abnormal neurological signs in the legs were found in all of them, and in 10 of the 14 reported there was gross muscle wasting. Loss of vibration sense occurred in all, and loss of proprioception and rombergism was noted in the majority. In those patients studied by muscle biopsy microscopical abnormalities of the terminal motor innervation were recognized as similar to those associated with nutritional neuropathy in coeliac disease of adults. $^{8}$ Only three of the patients responded well to vitamin $B_{12}$, and in them the clinical findings were typical of subacute combined degeneration of the cord. The neurological disease in the others may have been more like that which complicates adult coeliac disease, ${ }^{9}$ the aetiology of which is unknown.

The importance of recognizing the significance of symptoms in these rare patients is obvious. Unfortunately mental disturbances may increase the difficulties of recognition and management. Mental symptoms have been associated with vitamin $\mathbf{B}_{12}$ deficiency in some patients, and " psychopathic disturbances" were also a feature of several of the patients unresponsive to vitamin $B_{12}$ in the Birmingham series. ${ }^{2}$

\section{Prolonged Pregnancy}

About one pregnancy in ten is prolonged beyond the 42 nd week, and in such cases perinatal mortality is nearly double that at $37-41$ weeks. ${ }^{1-5}$ This is largely the result of a progressive inability of the placenta to support fetal nutrition and oxygenation in late pregnancy. Even in otherwise normal pregnancies hypoxia may reach a critical level by the end of the $42 \mathrm{nd}$ week. Prolonged pregnancy is also associated with an increased incidence of difficult labour. Birth trauma, pulmonary disorders, and failure to thrive create problems for the paediatrician-M. A. Zwerdling ${ }^{4}$ showed that the mortality rate in post-term infants was higher than average for at least the first two years of life, with the "small for dates" baby at particular risk. Surprisingly, no long-term sequelae have yet been found, as judged by studies of growth and intelligence.
Recently, N. A. Beischer and his colleagues ${ }^{5-7}$ have made an extensive review of the problems of prolonged pregnancy. In particular they have reassessed the value of urinary oestriol assays and examination of the liquor amnii as prognostic indices. They confirmed earlier reports which showed a good correlation between falling oestriol levels and other clinical evidence of failing placental function. Oligohydramnios and meconium staining of the liquor were associated with placental insufficiency and a high incidence of fetal distress in labour. Retrospective studies such as these have added much to our knowledge of the nature and degree of risk associated with prolonged pregnancy, but for various reasons attempts to apply the same techniques to prospective assessment in the individual case have generally been less successful.

Not the least of the many problems facing the clinician is the estimation of the duration of pregnancy; for doubt may remain even after consideration of menstrual dates and cycle, uterine and fetal size, liquor quantity and quality, and radiological and biochemical data. However, in such an assessment those features which concern chronological maturity are less important than those which more specifically reflect feto-placental dysfunction. The commonly used methods of practical value in the assessment of fetal well-being antenatally and during labour were reviewed last year by $R$. W. Beard. ${ }^{8}$ He confirmed the value of oestriol assays, provided serial observations are possible, but questioned the value of amnioscopy as at present applied.

These techniques are relatively time-consuming, and this must limit their practical application. Few units have facilities for full assessment of all women with prolonged pregnancy, but at least those patients at exceptional risk can be singled out and given special attention.

What, then, of the majority of patients with post-dated pregnancies? After the early work of J. Walker and E. P. N. Turnbull $^{9}$ a liberal policy of induction of labour was pursued in many units, but the response in terms of fetal loss has been disappointing. ${ }^{10}$ Some consider the hazards of induction of labour to be greater than those of allowing the pregnancy to continue. ${ }^{11}$ However, this is likely to be so only if arbitrary chronological criteria are adopted and if clinical assessment and the performance of induction are delegated entirely to junior staff.

Even when selective induction of labour is practised the fetus is already at risk to some degree. In prolonged pregnancy fetal death may occur before labour commences, but the greatest hazard is associated with anoxia during labour, especially if mechanical problems supervene. If placental insufficiency is severe, induction of labour is unlikely to save

1 Walker, J., Fournal of Obstetrics and Gynaecology of the British Empire, 1954, 61, 162.

- Gibson, G. B., British Medical fournal, 1955, 2, 715.

- Browne, J. C. McC., foumal of the American Medical Association, $1963,186,1047$.

- Zwerdling, M. A., Pediatrics, 1967, 40, 202.

S Beischer, N. A., Brown, J. B., and Townsend, L., American fournal of Obstetrics and Gynecology, 1969, 103, 496.

- Beischer, N. A., Brown, J. B., Smith, M. A., and Townsend, L., American fournal of Obstetrics and Gynecology, 1969, 103, 483.

7 Beischer, N. A., Evans, J. H., and Townsend, L., American fournal of Obstetrics and Gynecology, 1969, 103, 476.

- Beard, R. W., Proceedings of the Royal Society of Medicine, 1968, 61, 1247.

- Walker, J., and Turnbull, E. P. N., Lancet, 1953, 2, 312.

10 Browne, F. J., British Medical fournal, 1957, 1, 851.

1 Gibberd, G. F., Lancet, 1958, 1, 64.

12 Huntingford, P. J., and Pendleton, H. J., fournal of Obstetrics and Gynaecology of the British Commonwealth, 1969, 76, 586.

1s Bishop, E. H., Clinical Obstetrics and Gynecology, 1968, 11, 1154.

14 Saling, E., Foetal and Neonatal Hypoxia, 1968. London, Arnold.' 
the fetus, and caesarean section may be necessary. Yet during labour the detection of fetal distress is often a relatively haphazard affair. Because of practical limitations clinical auscultation of the fetal heart sounds allows no more than a glimpse of the overall behaviour of the fetal heart. Methods which require no special technical skill are, however, now readily available for continuous monitoring of the fetal heart rate and uterine contractions. ${ }^{12}{ }^{13}$ The greatest advance in accurate assessment of fetal oxygenation was the introduction by E. Saling ${ }^{14}$ of fetal blood sampling and estimation of $p \mathrm{H}$, but if serious errors are to be avoided it is important that staff should be carefully instructed and practised in its use.

The loss of a normally developed, healthy, mature infant solely as the result of anoxia is a particular tragedy, and it is imperative that every woman with suspected prolongation of pregnancy be given the benefit of assessment and delivery in a specialist unit. Many will agree with J. C. McC. Browne's ${ }^{3}$ conclusion that for a child to sail unscathed between the Scylla of postmaturity and the Charybdis of interference specialist skill of the highest order is needed.

\section{Pulmonary Sensitivity to Nitrofurantoin}

A seldom quoted side-effect of the drug nitrofurantoin is an acute eosinophilic pulmonary reaction which is almost certainly allergic in nature. ${ }^{1-3}$ Allergic side-effects of drugs should be examined in an immunological context. Other side-effects of nitrofurantoin have been many ; megaloblastic anaemia, haemolytic anaemia, aplastic anaemia and agranulocytosis which are relatively uncommon; and polyneuritis which is reported as frequent in patients with impaired renal function. These effects are probably toxic in origin.

Pulmonary infiltration on the other hand is very likely to be an immune response. It has certain features which suggest it might be a type $3^{4}$ or Arthus type reaction, but no confirmation of this has been obtained. If this were so, unrecognized cases could eventually proceed to pulmonary fibrosis. In fact, this long-term effect has now been reported in five patients. $^{5}$

The classic type 3 pulmonary reaction is seen in " pituitary snuff lung" which is due to the local effect of inhaled organic dust producing an allergic alveolitis with perivascular infiltration and, eventually, pulmonary fibrosis. Pulmonary vasculitis also occurs in systemic lupus erythematosus following hydrallazine, and systemic lupus erythematosus with pulmonary infiltration has been reported with isoniazid, tetracyclines, sulphonamides and iodides. ${ }^{7}$ These reactions are also thought to be type 3 allergic responses. Type 3 allergic responses are also involved in serum-sickness-like syndrome induced by drugs. Polyarteritis and vasculitis both acute and chronic have followed the use of penicillin, sulphonamides, and thiouracils. ${ }^{8}$ Polyarteritis nodosa may present as a pulmonary eosinophilia. Stevens-Johnson syndrome following, particularly, long-acting sulphonamides has also affected the lungs, producing pneumonic lesions and pleural effusion, ${ }^{9}$ and all these drug-induced conditions may possibly have a similar immunological basis.

The pulmonary sensitivity reaction to nitrofurantoin was nirst described by H. L. Israel and P. Diamond, ${ }^{10}$ who were able to show a direct cause-and-effect relationship, with a decreasing time interval between the administration of the drug and the appearance of the reaction with each succeeding course of treatment. There was also a progressively rising peripheral eosinophilia under these conditions.

Some 24 cases of pulmonary reactions to nitrofurantoin have now been reported. The fundamental lesion seems to be an acute eosinophilic infiltration with oedema of the lungs. Histology has shown airspaces packed with histiocytes and sheets of eosinophils in a protein-rich oedema ; there is perivasculitis but the interstitial tissue is otherwise unaffected.

The symptoms are sudden severe dyspnoea and high fever, and there is frequently myalgia and a maculopapular rash. Crepitations may be heard in the lungs, but there is no wheezing. Diffuse pulmonary shadows are seen on the radiograph in many cases, and pleural effusion may be present. Leucocytosis in the region of 12,000 to 15,000 neutrophils/ c.mm. may be seen initially, but if the drug is continued a very high eosinophilia may supervene (up to $10,000 /$ c.mm.). This sensitivity reaction may not occur with the first course of treatment, but if sensitization has been initiated the symptoms may develop fully within about eight hours of starting the second course. Sensitization may even develop during the first course of treatment, and the symptoms will then usually appear within seven to ten days. No fatalities have been reported, and there is a good response to treatment with corticosteroids. After withdrawal of the drug the condition resolves spontaneously within about 24 hours.

The pulmonary reaction to nitrofurantoin has many features in common with the transient eosinophilic lung infiltrations seen occasionally during treatment with P.A.S., ${ }^{11}$ penicillin, ${ }^{12}$ mephenesin, ${ }^{13}$ sulphonamides, ${ }^{14}$ and imipramine ${ }^{15}$; but in contrast to these the onset and course of the nitrofurantoin reaction appears to be more acute and severe. Difficulties have arisen over diagnosis, and the condition has been confused with acute pulmonary oedema of cardiac origin and also with pneumonia.

Awareness that the pulmonary reaction to nitrofurantoin may be more common than has previously been appreciated may reveal further cases of a less acute nature, and may also lead to prompt diagnosis and immediate withdrawal of the drug.

1 Nicklaus, T. M., and Snyder, A. B., Archives of Internal Medicine, 1968, 121, 151.

2 Allen, R. W., Holt, A. H., and Brown, M. G., American fournal of Roentgenology, Radium Therapy and Nuclear Medicine, 1968, 104, 4785 .

3 Brander, L., and Selroos, O., Acta Medica Scandinavica, 1969, 185, 215.

4 Gell, P. G. H., and Coombs, R. R. A., Clinical Aspects of Immunology, Oxford, Blackwell, 1968.

5 Rosenow, E. C., De Remee, R. A., and Dines, D. E., New England fournal of Medicine, 1969, 279, 1258.

- Pepys, J., Jenkins, P. A., Lachmann, P. A., and Mahon, W. E., Clinical and Experimental Immunology, 1966, 1, 377.

7 Assem, E. S. K., Hospital Medicine, 1967, 2, 199.

8 Symmers, W. St.C., Proceedings of the Royal Society of Medicine, 1962, 55, 20 .

- Carol, O. M., et al., in Drug Induced Diseases, Vol. III, edited by L. Meyler and H. M. Peck, p. 165, Amsterdam, Excerpta Medica . Meyler and 1968 .

10 Israel, H. L., and Diamond, P., New England fournal of Medicine, 1962, 266, 1024.

1 Wold, D. E., and Zahn, D. W., American Review of Respiratory Diseases, 1956, 74, 445.

12 Reichlin, S., Loveless, M. H., and Kane, E. G., Annals of Internal Medicine, $1953,38,113$.

13 Rodman, T., Fraimow, W., and Myerson, R. M., Annals of Internal Medicine, $1958,48,668$.

${ }^{14}$ Fiegenberg, H. W., Weiss, and Krishman, H., Archives of Internal Medicine, 1967, 120, 85 .

15 Wilson, I. C., Gambill, J. M., and Sandifer, M. G., American fournal of Psychiatry, 1963, 119, 892. 